\title{
Hemostasis Laboratory Diagnostics: Characteristics, Communication Issues, and Current Challenges Resulting from Centralization of Laboratory Medicine
}

\author{
Rüdiger E. Scharf ${ }^{1}$ \\ 1 Program in Cellular and Molecular Medicine, Boston Children's \\ Hospital, Harvard Medical School, Boston, Massachusetts, USA \\ Hämostaseologie 2020;40:403-412.
}

\begin{abstract}
Address for correspondence Rüdiger E. Scharf, MD, PhD, FAHA, Program in Cellular and Molecular Medicine, Boston Children's Hospital, Harvard Medical School, Karp Family Research Laboratories, 1 Blackfan Circle, RB09211.1, Boston, MA 02115, USA (e-mail: ruediger.scharf@childrens.harvard.edu).
\end{abstract}
Abstract
Keywords
- laboratory testing
- hemostasis
- diagnostic algorithms
- quality control
- communication

\begin{abstract}
Laboratory diagnostics of patients with bleeding and thrombotic disorders can be a delicate task, which requires special skills and expertise. In this article, characteristic features of hemostasis testing are reviewed, including staged protocols and synoptic assessment of the patient history, clinical symptoms, and laboratory findings. Despite major progress in the diagnostic and therapeutic management, centralized testing of hemostasis can be associated with substantial challenges, resulting from the current dissociation between the clinical and laboratory world. To address some of these challenges, possible solutions are discussed, including adaptation of an established working paradigm.
\end{abstract}

\section{Introduction}

In approximately 60 to $70 \%$ of patients, medical laboratory results directly contribute to diagnosis and disease management, including follow-up examinations, monitoring of therapy, and assessment of treatment-associated or, specifically, drug-induced side effects. ${ }^{1}$ The qualitative and quantitative impact of laboratory testing is even higher when subjects participating in prevention, screening, or early diagnosis programs are also enrolled. Due to its essential role, the clinical laboratory has been defined as "the nerve center of diagnostic medicine."

With regard to hereditary or acquired bleeding and thrombotic disorders, staged protocols of appropriate laboratory testing are indispensable to identify the defect or dysfunction in a given patient, specifically to define and classify the type of an underlying disorder, to assess the effect of hemotherapy, and to monitor anticoagulant, antiplatelet, fibrinolytic, or antifibrinolytic treatment.

\section{Progress in Laboratory Diagnostics}

Over the past few decades, we have witnessed significant advancements in laboratory medicine. Key elements of these advancements are technical and methodological inno- vations such as larger, faster, and more efficient laboratory instruments, including high-speed analyzers, total laboratory automation, and new information technologies. ${ }^{1}$ In combination with novel biochemical, molecular, and genetic biomarkers, the volume of samples to be processed and analyzed has enormously increased. Simultaneously, analytical state-ofthe-art instruments, automation, and information technology have shortened the turnaround time and allow reporting of real-time results for a large proportion of laboratory test requests in a round-the-clock mode ("24-7-365" service). Implementation of internal quality control and external quality assurance programs in accord with national and/or international regulatory requirements have improved test reliability and substantially reduced analytical errors. ${ }^{1,3,4}$

Similar progress has also been made in laboratory testing of hemostasis and thrombosis. Specifically, the discovery and introduction of novel diagnostic and prognostic biomarkers into practice have greatly stimulated the management of patients with hemostatic disorders.

\section{Centralization of Medical Laboratories}

Along with advances in science and technology (e.g., novel biomarkers, innovative diagnostic assays, next-generation received

September 30, 2020

accepted after minor

revision

October 2, 2020 (c) 2020 Georg Thieme Verlag KG Stuttgart · New York
DOI https://doi.org/ 10.1055/a-1249-8767. ISSN 0720-9355. 
sequencing), translational research (e.g., "omics" diagnostics such as genomics, proteomics, or metabolomics), and infrastructural improvements (automation, information technologies) during recent decades, economic stimuli and current financial constraints have been essential drivers for laboratory centralization of clinical pathology, including traditional clinical chemistry, immunochemistry, and laboratory hematology. As a consequence of this evolution, in the majority of university and large community hospitals, laboratory diagnostics of hemostasis and thrombosis have also been integrated into the central medical laboratory, in most institutions under the leadership of a professional in clinical chemistry and laboratory medicine.

Typical features of centralized testing in core laboratory facilities are large series of analyses ("the bigger the better"), one-to-one handling, processing and analysis of parameters, as ordered by the clinical "customer", automated calibration and validation of instruments or procedures, automated test systems and analyses, short turnaround times ("the faster the better"), internal quality control, external quality assurance of analyses, and automated reporting of laboratory test results.

\section{The Brain-to-Brain Loop Concept: A Working Paradigm}

According to the traditional Lundberg concept of the "brainto-brain turnaround time loop" ${ }^{5}$, the generation of any laboratory test result consists of nine steps: (1) selection of test parameter and ordering, (2) specimen collection (serum, EDTA-, or citrate-anticoagulated blood), (3) identification (at several stages), (4) transportation and/or storage, (5) separation (or preparation), (6) analysis, (7) reporting, (8) interpretation, and (9) action. ${ }^{3}$ Accordingly, preanalytical (steps 1 through 5), analytical (step 6), and postanalytical phases (steps 7 through 9) are defined (-Fig. 1). Data analysis, interpretation of results, clinical consequences, and outcomes are essential issues of the working paradigm.

Usually, with regard to basic hematology, clinical chemistry, and immunochemistry parameters, the vast majority of laboratory results is typically neither commented nor interpreted through laboratory staff physicians. By contrast, interpretation of results is left to the clinicians themselves, thereby referring to defined reference ranges of normal values. Thus, in clinical practice, the central laboratory more or less functions as a core facility providing services in generating data ("values") but no corresponding solution approach or direct answer to clinical issues. Moreover, current economic compulsions and constraints have moved the focus of central laboratories in the direction asking "What is the cost (or revenue) per tested parameter or reported result, respectively", but not addressing the essential question "What is the benefit for the patient care?"

By contrast to routinely performed analyses in clinical chemistry, immunochemistry and basic hematology, laboratory testing of hemostasis is much more delicate. Thus, laboratory hemostasis remains a challenging field both for clinicians and laboratory professionals unless specifically trained and experienced. Importantly, the appropriate diag- nostic workup of bleeding or thrombotic disorders requires a comprehensive synopsis of the patient history, symptoms, and laboratory findings. This synopsis is hard if not even impossible to achieve in a central laboratory "factory", in which clinical and laboratory demands are most commonly dissociated.

\section{Specific Features of Laboratory Hemostasis and Thrombosis}

Hemostasis is a dynamic biological system, involving distinct players and complex, multifaceted processes. Consequently, laboratory analysis of the cellular and plasma components and/or multiple pathways is a challenging task both in health and disease.

Unlike other areas of laboratory medicine and clinical pathology, laboratory hemostasis has some special characteristics, including the need of anticoagulated blood samples as biological matrix (for platelet testing and coagulation or fibrinolysis assays) and also the requirement of performing more than one assay to achieve a final diagnosis (see below). Along with that, specimens for hemostasis testing are particularly vulnerable to preanalytical variables and errors. ${ }^{4}$ For example, improper filling of primary collection tubes and incorrect mixing of blood with the preloaded anticoagulant (i.e., buffered sodium citrate at a final concentration of $3.2 \%$ ) may ultimately lead to erroneous test results. Preanalytical errors can account for approximately $70 \%$ of errors in laboratory testing. ${ }^{1}$

Another specific feature of laboratory hemostasis is that staged procedures are needed to confirm or exclude a suspected hemostatic disorder in most clinical settings and individual patients. However, unlike other areas of laboratory medicine, standardization (e.g., platelet aggregometry), harmonization of certain diagnostic algorithms, and even harmonization of test procedures (e.g., von Willebrand factor [VWF] analysis) are not always met in hemostasis laboratory practice. ${ }^{6}$

\section{Principles of Laboratory Hemostasis Testing}

Laboratory analysis of hemostasis should typically follow a stepwise procedure. ${ }^{7}$ Routinely performed baseline assays (step 1) include the activated partial thromboplastin time (aPTT), prothrombin time (PT; “Quick"; international normalized ratio), fibrinogen (Clauss), D-dimers, and platelet count but not platelet function. Different from these "basic" tests are screening assays (step 2) to answer one of the following questions: (1) Is there any laboratory indication of a hemostatic disorder? (2) Which component of the hemostatic apparatus may be affected or compromised? (3) In which direction the laboratory diagnostic should be extended?

In addition to step 1, screening assays encompass assessment of platelet function by determination of in vitro bleeding time (platelet function analyzer-100 [PFA-100]), evaluation of VWF parameters (VWF activity and VWF antigen), factor XIII activity, screening for lupus anticoagulant (LA) by at least two assays (e.g., LA-sensitive aPTT and dilute Russell viper venom test), and screening for increased fibrinolysis (D-dimers). Global assays of hemostasis such as thrombelastography and thrombin generation assay may 


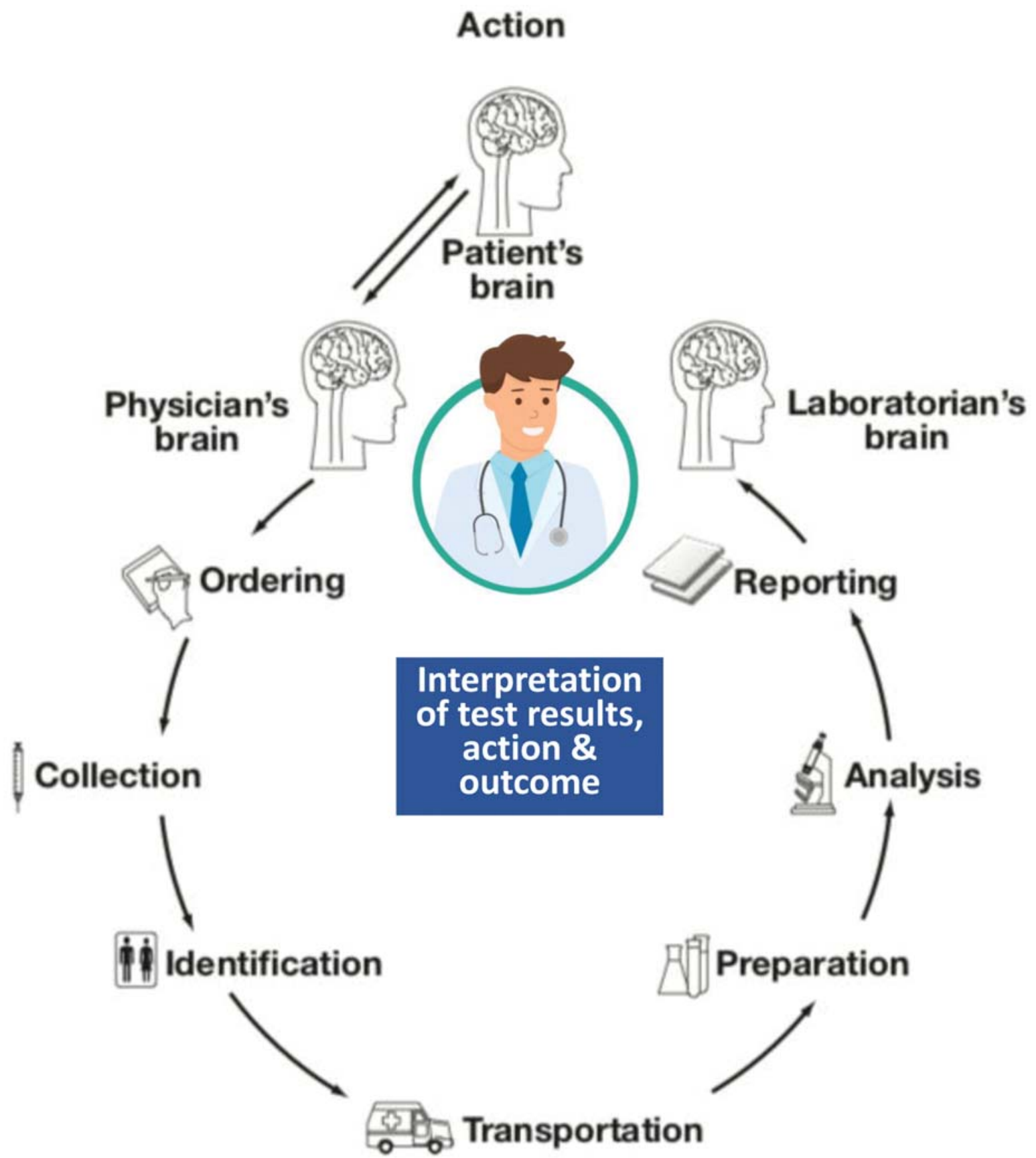

Fig. 1 Adaptation of Lundberg's "brain-to-brain loop" concept for laboratory testing. The traditional concept ${ }^{5}$ (as partly shown here in black and white) represents a working paradigm designed to define the physician-laboratory interaction and the physician-patient relationship. According to this concept, the generation of any laboratory test result consists of nine steps, ranging from selection of test parameters and ordering (step 1) to proactive clinical consequences (step 9, originally designated "action"). Nowadays, the dissociation and dichotomization between the "clinical world" and the "laboratory world" with progressive autonomy in the production of laboratory results represents a fracture of the original brain-to-brain loop model and can have a negative impact on patient care (and outcome), particularly in patients with hemorrhagic or thromboembolic disorders. The physician (depicted in color) holds a central position within this circle and exercises pivotal functions, as outlined in the text. Ideally, the physician is specifically qualified and experienced in hemostasis and thrombosis and capable of actively bridging the current gap between clinical and laboratory medicine. The relevance of the physician's central role is also evident by the fact that current centralized laboratory testing of hemostasis is often encumbered by decreased or even missing interaction between clinicians and laboratorians. (Modification of a scheme, taken from Plebani et al. ${ }^{3}$ )

be useful additional tests at this stage but have not found widespread application in daily practice. ${ }^{8}$

Step 3 comprises problem-oriented detailed analytics such as mixing tests, coagulation factor activities and/or single-function assays (e.g., platelet aggregometry, ristoce- tin-induced platelet agglutination) to provide further insights into abnormalities of screening tests or to monitor antithrombotic treatment in a more accurate way. In case of clinically suspected or evident thrombophilia, antithrombin, proteins $\mathrm{C}$ and $\mathrm{S}$, resistance to activated protein $\mathrm{C}$, thrombin- 
antithrombin and plasmin-antiplasmin complexes are also tested at this stage. Confirmation of LA requires determination of anticardiolipin and anti- $\beta 2$-glycoprotein-I IgM and IgG antibodies.

Molecular genetic analyses to identify polymorphisms or mutations of distinct coagulation factors (e.g., factor V Leiden, prothrombin G20210A), fibrinolytic components (e.g., plasminogen activator inhibitor-1 [PAI-1] with 4G/5G insertion/deletion polymorphism at position -675 in its promotor), or of platelet adhesion or aggregation receptors (e.g., GPIb-IX-V, $\alpha$ IIb $\beta 3, \alpha 2 \beta 1)$ are reserved to another stage of diagnostics (step 4).

While most of these assays (steps 1 through 4) are established in the majority of clinical laboratories, further testing and analyses (step 5) using protein chemical, immuno- and flow cytometric, or distinct functional techniques are left to specialized hemostasis centers. Thus, the precise classification of von Willebrand disease (VWD) requires multimer and molecular analyses. Other settings include the detection, quantitation, and monitoring of coagulation factor inhibitors (e.g., in acquired hemophilia A), analyses of rare thrombophilic mutations, rare platelet functional disorders, or pharmacogenetics testing. This hierarchy is by no means standardized. Thus, others have simplified the hierarchy of staged hemostasis testing by condensing steps 1 and 2 (firstline tests for screening), steps 3 and part of 4 (second-line tests for etiological diagnosis), and part of step 4 plus step 5 (third-line tests for biochemical or molecular characterization of the alteration). ${ }^{6,8}$

In accord with the stepwise analytic procedures (stages 1 through 5), algorithms exist that guide the testing pathway and allow a rational diagnostic workup. These algorithms encompass both bleeding ${ }^{9-16}$ and thrombotic ${ }^{15,17-22}$ disorders or abnormal findings in laboratory hemostasis. For example, in case of a prolonged aPTT, a rather frequent and multicausal finding in hemostasis laboratory diagnostics, ${ }^{23}$ the algorithms direct the type and sequence of tests to permit a clear differential diagnosis and to identify the precise cause of the abnormality, including rare clinical conditions. ${ }^{24-26}$

\section{Preoperative or Preinterventional Hemostasis Screening}

Numerous studies exist reporting on failure, uselessness, insensitivity, or missing specificity of laboratory tests to predict intra- or postoperative bleeding in different patient cohorts undergoing surgery or other interventions. ${ }^{27-35}$ Several investigators have also assessed the cost-effectiveness and the putative impact of laboratory diagnostics on clinical decision-making and outcome in this context. ${ }^{30,34}$

Some of these studies appear to be biased. ${ }^{27-29,31-34}$ For example, when using baseline parameters such as aPTT, PT, fibrinogen, and platelet counts only ("step 1"), one can easily anticipate that this test panel is inappropriate to identify the majority of patients with increased bleeding risk. Conversely, a broad spectrum of hemostasis assays in unselected patients is generally not indicated in the preoperative or preinterventional setting but wasting time and laboratory and/or clinical resources. In addition, given the low prevalence of bleeding disorders in the general population and the wide range of possible clinically irrelevant alterations (e.g., prolonged aPTT due to FXII deficiency), the positive predictive value of a pathologic result in screening hemostasis is extremely low.

\section{Clinical Aspects and Frequency of Hemostatic Defects} Remarkably, "pure" coagulation defects occur less frequently than generally assumed in clinical practice. This conclusion is derived from a prospective study by Koscielny et al, who studied more than 5,600 consecutive patients prior to elective surgery. ${ }^{36}$ Patients with known preexisting hemostatic defects or anticoagulation therapy were primarily excluded from the analysis. Of the total cohort, 628 patients (11\%) had a bleeding history, and laboratory screening for a hemostatic defect was positive in 256 of them (40.8\%). Interestingly, diagnostic workup of these 256 subjects revealed platelet dysfunction in 187 (73\%), coagulation disorders in 2 (0.8\%), and combined hemostatic defects in 67 patients (26.2\%), including a predominant proportion of patients with VWD. ${ }^{36}$ Among the 187 individuals with defects in primary hemostasis, acquired platelet dysfunction was drug-induced in 162 of 256 patients (63.3\%).

\section{Conclusions from the Study}

The data reported by Koscielny et $\mathrm{al}^{36}$ illustrate several important issues: (1) acquired platelet function defects are more frequent in clinical practice than generally believed; (2) in the majority of cases, platelet dysfunction is drug-induced; (3) among congenital hemostatic disorders, VWD must not be underestimated; and (4) "pure" coagulation defects, as documented here (in 2 of 256 patients), are less frequent in unselected adult subjects than commonly estimated. ${ }^{37,38}$

\section{Consequences}

Accordingly, screening for primary hemostasis defects requires indeed useful laboratory tools, as demonstrated by the PFA technique. Testing of closure times is particularly sensitive to VWF anomalies ${ }^{11,39}$ and has largely replaced the determination of the in vivo bleeding time by the susceptible and poorly reproducible Ivy method (Simplate I or II device). Albeit trivial, the widespread practice of platelet counting and coagulation screening (by baseline assays of "step 1", as indicated earlier) is entirely inappropriate to identify individuals with platelet dysfunction due to an acquired platelet defect and/or inherited VWD. ${ }^{37,38}$

A more general critique of the above "screening" assessment studies is that some investigations disregard that laboratory analysis is only one of several elements in the diagnostic workup. Importantly, both the patient's detailed history, specifically bleeding or thrombotic complications during previous interventions or surgical trauma, and careful physical examination remain the cornerstones of the diagnostic strategy both for bleeding and thrombotic disorders.

\section{Drug-Induced Platelet Dysfunction}

Drugs represent the most common cause of acquired platelet dysfunction in our overmedicated society. The list of agents 
that can affect platelet function is extensive and not restricted to typical antiplatelet drugs. ${ }^{40}$ Therefore, evaluation of the past and present medication history (including over-thecounter agents and herbal remedies) is essential for diagnosis, management, and prevention. However, meticulous assessment, recording, and evaluation can be laborious and time-consuming and are therefore not popular among busy clinicians.

\section{Clinical Examination}

Comprehensive physical examination and correct interpretation of clinical symptoms can also provide relevant information to direct the problem-oriented laboratory diagnostics of hemostasis. Thus, in patients with hemorrhagic diathesis, the skin bleeding phenotype can be indicative of the hemostatic component that is most likely affected. For example, sharply demarcated hematomas typically occur in coagulation defects, whereas petechiae and cloudy bruises are suggestive of platelet dysfunction, relevant thrombocytopenia, and/or vascular disorders 41 (-Fig. 2). In senile purpura, chronic corticosteroid treatment or Cushing's syndrome, bruising and cutaneous bleeds occur after minor trauma, or even spontaneously, without systemic hemorrhage. There are several other conditions, in which the hemostatic system is not compromised. Typical diagnostic signs include bilateral periorbital ecchymoses (raccoon's eyes), indicative of light-chain amyloidosis ${ }^{42}$, and cutaneous telangiectasia pathognomonic of Morbus Osler-Weber-Rendu, nowadays designated hereditary hemorrhagic telangiectasia. ${ }^{43,44}$ In all these settings, extensive but fruitless laboratory hemostasis testing should be avoided.

Regarding venous thromboembolism, clinical features are equally fundamental but less clearly to interpret due to the heterogeneity of suggestive symptoms.

\section{Current Recommendations and Guidelines}

To assess the bleeding and the thromboembolic risk, more recent recommendations ${ }^{10,45-49}$ and national or international guidelines ${ }^{50-53}$ have stressed the need for careful and complete evaluation of the patient's history and also emphasized the importance of a thorough clinical examination. However, this requirement is frequently not met in reality, as evident outside of studies, in daily clinical practice.

To overcome this dilemma and to increase both the diagnostic efficiency and the patient's safety, specific questionnaires combined with scoring systems (bleeding assessment tools) have been introduced and validated. ${ }^{13,54-58}$ However, this approach has major limitations and does not meet the high expectations regarding the discriminating power in the preoperative setting. For example, a guideline-based questionnaire cannot differentiate between
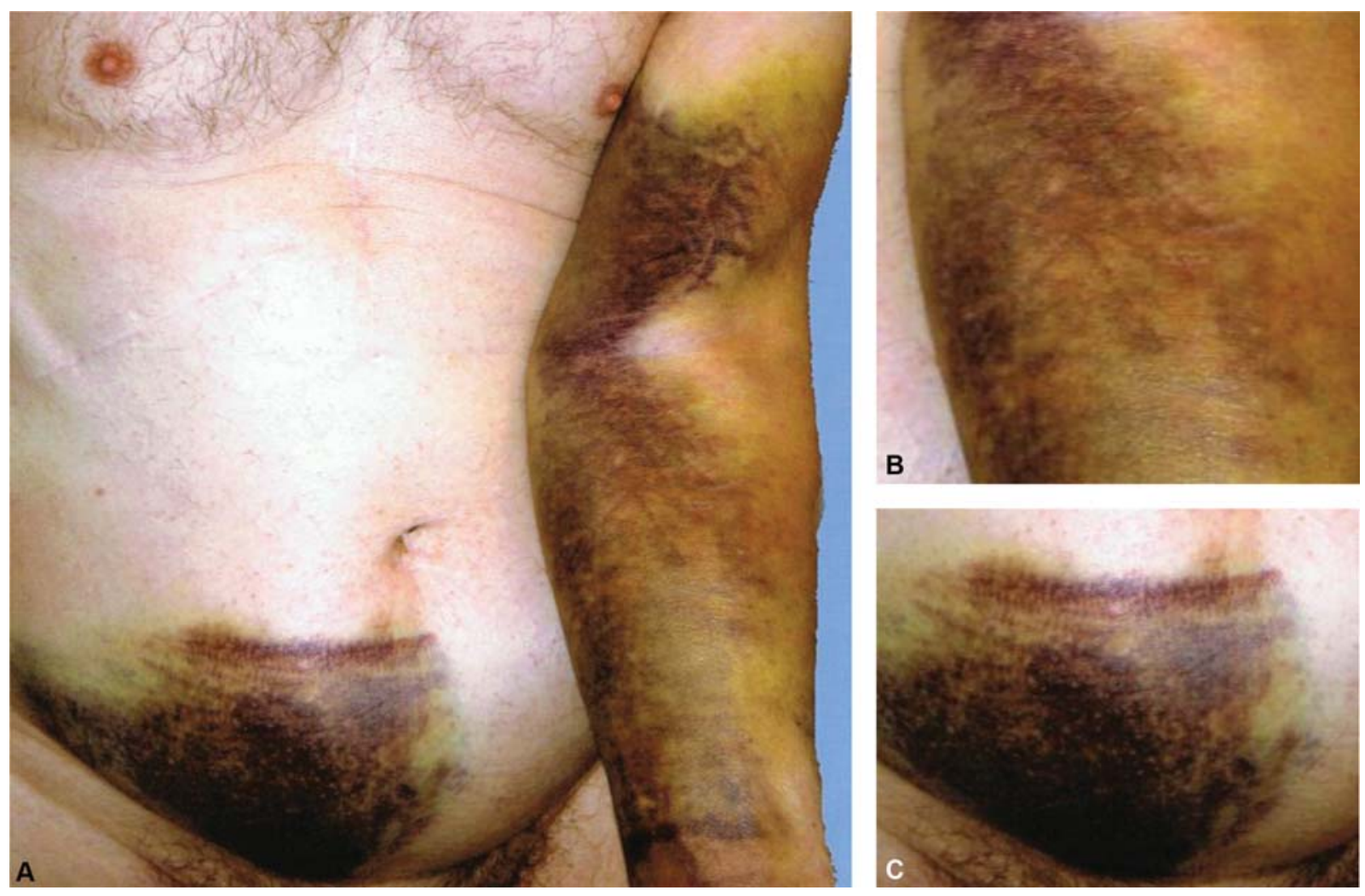

Fig. 2 Distinct bleeding phenotypes in a patient on combined antithrombotic treatment. (A) The patient experienced cutaneous bleeds after receiving dual-antiplatelet therapy (aspirin plus clopidogrel) in combination with anticoagulation (heparin) because of suspected acute coronary syndrome. Of note is the different bleeding phenotype displaying (B) "cloudy bruises" (typical of platelet dysfunction, thrombocytopenia, and/ or vascular disorders) at the flexor side of the arm and (C) a sharply demarcated abdominal hematoma (typical of coagulation disorder or side effect of anticoagulation). Thus, careful clinical assessment can provide an indication of which hemostatic component might be affected and allow targeted screening of either platelet/vascular or coagulation defect(s). (Modification, taken from Scharf. ${ }^{41}$ ) 
patients with and without reported bleeding symptoms, as demonstrated recently. ${ }^{58}$

\section{Centralization and Communication at the Laboratory-Clinical Interface}

The current organization and operational performance of laboratory medicine, including extensive use of automation, other mass-production techniques, and information technologies, have enabled operators to guarantee a timely release of validated laboratory results despite the enormous increase in test requests and the resulting workload due to regulatory requirements and the application of quality management tools. However, structural and organizational changes have created a dichotomy between the clinical and laboratory world. This evolution is accompanied by several pitfalls at the laboratory-clinical interface. Currently unresolved or incompletely accomplished issues in hemostasis and thrombosis testing are summarized in -Table 1. For example:

1. The dissociation between clinical demands and laboratory processing does not allow a careful and competent incoming control of each requested assay or parameter in a given patient.

2. The progressive autonomy from the clinical context can frequently be associated with inappropriate test requesting. Given the fact that the selection of assay parameters at the bedside is often performed by young physicians in training, who have poor knowledge in hemostasis, lacking incoming controls of test orders on-site are a major concern.

3. Incomplete (or even missing) clinical information about the patient history, medication (e.g., antiplatelet or anticoagulant agents), and relevant symptoms upon physical examination is another frequent failure that can lead to incorrect interpretation of laboratory results. For example, pregnancy-associated upregulation of coagulation and fibrinolysis parameters would be misinterpreted and may be considered "abnormal" without the information "pregnancy."

4. Specifically, a clinical question defined as precisely as possible is required to perform a problem-oriented and targeted laboratory hemostasis testing in accord with stepwise procedures, as outlined in section "Principles of Laboratory Hemostasis Testing."

An article by Kemkes-Matthes is underway, reporting on various scenarios and settings that illustrate to which extent missing or incomplete clinical information can bias laboratory hemostasis testing or cause misinterpretation of assay results. ${ }^{59}$ It should be emphasized that laboratory order forms structured according to different clinical settings (e.g., bleeding or thrombotic diathesis, preoperative screening, monitoring of antithrombotic treatment) may be useful tools. However, such forms cannot compensate for proper communication at the laboratory-clinical interface or replace mutual consultations between clinicians and laboratorians.

Table 1 Currently unresolved or incompletely accomplished issues in hemostasis testing at the interface between clinic and centralized laboratory

\begin{tabular}{|l|}
\hline Poor knowledge of hemostasis in health and disease among clinicians and laboratory professionals \\
\hline Awareness and harmonization of preanalytical, analytical, and postanalytical procedures \\
\hline Definition of precise questions to be addressed from the clinician to the laboratorian \\
\hline Selection of corresponding hemostasis parameters or test panels \\
\hline $\begin{array}{l}\text { Incoming control of requested test parameters, check of consistency and completeness, and prevention of over- or under- } \\
\text { diagnosing in a given setting or clinical condition, downsizing or extension of requested test panels in individual cases }\end{array}$ \\
\hline Heterogeneity of available guidelines for diagnosis and/or therapeutic management \\
\hline Heterogeneity of diagnostic algorithms for laboratory testing \\
\hline Inaccurate definition of reference ranges ${ }^{6}$ of several hemostasis parameters in certain clinical conditions (e.g., pregnancy) \\
\hline Identification and rapid reporting of "true" critical values ${ }^{6}$ \\
\hline Communication of complete clinical information about the patient's history and condition \\
\hline Correct interpretation of laboratory test results by synopsis of patient history, symptoms, and laboratory findings \\
\hline $\begin{array}{l}\text { Integration of clinicians into laboratory issues and, vice versa, participation of laboratorians in clinical activities (e.g., } \\
\text { attending ward rounds, mutual consultations) }\end{array}$ \\
\hline Assessment of clinical outcome through laboratory testing and feedback to the laboratorian \\
\hline $\begin{array}{l}\text { Implementation of "integrated" cost analysis (and reimbursement), i.e., cost per hospital stay and total patient care, not } \\
\text { cost per reported result }\end{array}$ \\
\hline $\begin{array}{l}\text { Training programs of medical students and continuing medical education (CME) of clinicians and laboratory staff in } \\
\text { hemostasis and thrombosis }\end{array}$ \\
\hline Implementation of ISTH core curricula for clinical ${ }^{73}$ and laboratory ${ }^{74}$ hemostasis and thrombosis specialists \\
\hline
\end{tabular}




\section{Point-of-Care or Near-Patient Testing of Hemostasis}

In response to the centralization of medical laboratories, their "factory" performance and "insular" existence, and, in particular, in response to the more and more increasing dichotomy and diversity between the "clinical world" and the "laboratory world," a new generation of point-of-care testing (POCT) or near-patient testing (NPT) instruments has been developed in recent years. These easy-to-use analytical devices are applied for screening of global hemostasis (e.g., thrombelastography), coagulation and fibrinolysis disorders (e.g., ROTEM), and/or platelet function (e.g., PFA-200; VerifyNow System; Multiplate Electrode Aggregometry; Impact Cone and Plate Analyzer) in the emergency, intensive care, perioperative or interventional, and even outpatient setting. ${ }^{60-67}$ Application of POCT reduces turnaround times, thereby facilitating rapid availability of screening results that in turn can enable prompt diagnostic or interventional decisions. In fact, NPT under laboratory governance can bridge the gap between bedside patient care and laboratory medicine. However, stringent quality control management of POCT instruments and assays is a relevant concern, ${ }^{61}$ and most laboratorians consider POCT as an "alien" or "dangerous alternative" to traditional laboratory medicine in a core facility. ${ }^{1}$

\section{Adaptation of the Brain-to-Brain Loop Concept on Centralized Hemostasis Testing}

In accord with the traditional brain-to-brain loop concept, the much-invoked synopsis of patient history, symptoms, selection of hemostasis tests or test panels, analysis, and test result interpretation was easier to achieve under guidance of an experienced clinical specialist in hemostasis and thrombosis, who had also responsibility of the hemostasis core laboratory. Unfortunately, this personal union has become exceptional in most academic institutions. In fact, as discussed earlier, the structural and organizational dichotomization between clinical and laboratory responsibility represents a fracture of the original brain-to-brain loop model. Consequently, the traditional concept requires adaptation on centralized testing in hemostasis and thrombosis. Such an adaptation is shown in -Fig. 1. The physician depicted in the center acts as hemostasis and thrombosis consultant and has to fulfill several crucial tasks by:

1. Defining (or confirming) a precise clinical question addressed to the centralized laboratory.

2. Selecting appropriate tests (or test panels).

3. Providing the earlier-indicated synopsis of patient-related features and assay results to allow correct interpretation of test results (or extend laboratory investigations if indicated according to staged protocols or algorithms).

4. Initiating a corresponding or problem-oriented clinical action (diagnostic and/or therapeutic management).

5. Evaluating the clinical outcome.
These comprehensive tasks will require long-standing expertise in hemostasis and thrombosis to bridge the currently existing gap between the clinical and laboratory world. Conversely, with regard to laboratory professionals, to regain their relevant role in diagnostics and fulfill their original mission, laboratory medicine and laboratorians need to be integrated (or reintegrated) into patient care pathways. ${ }^{1}$ However, it is evident that these demands describe conditions that will require a paradigmatic change. It remains to be seen whether or not such a change will occur.

\section{Distorted Economics by Inappropriate Testing in Centralized Laboratories}

To the author's knowledge, no study exists in which the real economic overall effect of hemostasis testing in a centralized medical laboratory has ever been evaluated or, at least, estimated reliably. Such an analysis would strictly require to include and carefully assess the expenditures of unnecessary or unwarranted laboratory test orders, false-positive or falsenegative test results (due to preanalytical, analytical, or postanalytical errors), as well as subsequent costs arising from prolonged hospital journeys or unfavorable clinical outcome.

Studies addressing the appropriateness of laboratory testing related to various clinical conditions in the hospital setting have reported an overutilization of approximately $20 \% .{ }^{68,69}$ However, reviews and meta-analyses can convey a rough idea of this issue in general only. More informative is a recent study by Sarkar et al, ${ }^{70}$ who assessed the proportion of diagnostic errors in the context of over- and underutilization of laboratory tests when patients were evaluated for bleeding or thrombotic disorders in a U.S. university medical center. Review of hemostasis test requests in real time by a panel of experts revealed $77.5 \%$ diagnostic errors ( 155 cases) among 200 randomly selected patients. Interestingly, $16 \%$ of the cases were associated with overutilization of laboratory tests, $44 \%$ with underutilization, and $17.5 \%$ with both. The annual cost burden generated by delay in diagnosis or misdiagnosis (in cases with underutilization) was approximately 220,000 USD in this small group of patients.

\section{Conclusion and Perspectives}

Hemostasis laboratory diagnostics will remain a delicate task. Until recently, specialists in hemostasis and thrombosis, equally well-trained in clinical and laboratory medicine, were guiding the laboratory diagnostic and therapeutic management in this field. This organizational and operational constellation facilitated problem-oriented testing along diagnostic pathways, targeted selection of test parameters, correct interpretation of test results, and appropriate clinical management.

Today, centralized testing and integration of hemostasis and thrombosis into clinical chemistry and laboratory medicine, mostly driven by increasing economic pressure and financial constraints, have created new challenges. In this context, the dissociation between the clinical 
world and the laboratory world is a major concern, whereas the laboratory-clinical interface was functional in the past. However, one cannot turn back the "clock of centralization."

The progressive autonomy of centralized laboratory factories under the leadership of laboratory (but not clinical) professionals has resulted in unfavorable developments. Thus, typical laboratory-related issues such as efficiency, productivity, timeliness, and regulatory and economic issues dominate the daily management of laboratorians, whereas their contribution to patient care has drifted out of focus. ${ }^{1}$ How can we improve the current situation under these circumstances?

First, it is worth remembering that the original mission of laboratory medicine is (and remains) to provide medical service to support patient care and improve outcome. Second, to react to the dichotomy between laboratory and clinical medicine and to overcome the gap between laboratorians and physicians at the bedside, a competent and experienced personality needs to be inaugurated, who is capable of acting as a "bridge builder" between two arenas (-Fig. 1). Some of the essential tasks have been outlined and discussed earlier. However, such individuals with comprehensive core competence in thrombosis and hemostasis, communication skills, and organizational qualification are rare among the acting generation. Possible solutions of this approach still lie ahead.

Third, it will be pivotal to make significant investments in the education and training of the young generation of medical graduates and physicians entering clinical and/or pathology specialties. Academic institutions and professional organizations should promote valuable training programs, provide continuing medical education, increase mentoring, and stimulate research activities. Specifically, in the field of hemostasis and thrombosis, attractive scientifically guided education and comprehensive training programs will be essential to augment the competencies and practice-related skills.

The Society of Thrombosis and Hemostasis Research (GTH) has addressed these challenges. Apart from educational sessions at the annual GTH congress, the GTH Academy offers several attractive training and education programs, including the long-established GTH Intensive Course on Clinical and Laboratory Hemostasis and the GTH Highlights. Recently, the society's academy has launched a new program, a media library covering relevant topics of the state-of-theart hemostasis. ${ }^{71}$ Moreover, the GTH has extended the society's portfolio of awards and now offers early career research grants for competitive funding of young scientist in hemostasis, thrombosis, vascular biology, or translational research. $^{72}$

Along with these GTH activities, the International Society on Thrombosis and Haemostasis (ISTH) has defined a set of core competencies for clinical specialists taking care of patients with disorders of thrombosis and hemostasis. ${ }^{73}$ Moreover, thrombosis and hemostasis laboratory specialists require distinct competences that differ from clinicians. Recently, the ISTH has also developed an evidence-based core curriculum for laboratorians. ${ }^{74}$ Both documents may be used for improvement and implementation of more standardized educational programs, future accreditation, or even formal assessment across jurisdiction.

Overall, the conceptual approaches and multiple activities of scientific societies, summarized here in part, are giving cause to hope that appropriate contributions of laboratory hemostasis and thrombosis testing will refocus attention on its original mission and main objective, which are patient care and outcome.

\section{Conflict of Interest}

None declared.

Acknowledgments

I am grateful to my colleagues for stimulating (and controversial) discussions on the changing laboratory-clinical interface. Work from my laboratory that is cited here was supported by grants from the Deutsche Forschungsgemeinschaft (Scha 358/3-1; Collaborative Research Center, SFB 612, Project B2). Funding of the author's research at Boston Children's Hospital by a GTH grant is also acknowledged.

\section{References}

1 Plebani M. Clinical laboratories: production industry or medical services? Clin Chem Lab Med 2015;53(07):995-1004

2 Cortelyou-Ward K, Rotarius T, Liberman A, Trujillo A. Hospital inhouse laboratories: examining the external environment. Health Care Manag (Frederick) 2010;29(01):4-10

3 Plebani M, Laposata M, Lundberg GD. The brain-to-brain loop concept for laboratory testing 40 years after its introduction. Am J Clin Pathol 2011;136(06):829-833

4 Favaloro EJ, Lippi G, Adcock DM. Preanalytical and postanalytical variables: the leading causes of diagnostic error in hemostasis? Semin Thromb Hemost 2008;34(07):612-634

5 Lundberg GD. Acting on significant laboratory results. JAMA 1981;245(17):1762-1763

6 Lippi G, Favaloro EJ. Hemostasis practice: state-of-the-art. J Lab Precis Med 2018;3:67

7 Scharf RE. Staged laboratory diagnostic protocols in hemostatic disorders. In: Scharf RE, Gerhardt A, eds. Hemostasis and Thrombosis, 8th ed. Heinrich Heine University Medical Center Document, Düsseldorf University Press; 2014:28-31

8 Lippi G, Favaloro EJ. Laboratory hemostasis: milestones in clinical chemistry and laboratory medicine. Clin Chem Lab Med 2013;51 (01):91-97

9 Kottke-Marchant K, Corcoran G. The laboratory diagnosis of platelet disorders. Arch Pathol Lab Med 2002;126(02):133-146

10 Pötzsch B, Scharf RE. Hemostasis-navigated hemotherapy: diagnosis, management, and monitoring of patients with active bleeding. In: Scharf RE, ed. Progress and Challenges in Transfusion Medicine, Hemostasis, and Hemotherapy. Basel: Karger; 2008:385-393

11 Greaves M, Watson HG. Approach to the diagnosis and management of mild bleeding disorders. J Thromb Haemost 2007;5 (Suppl 1):167-174

12 Rodeghiero F, Tosetto A, Castaman G. How to estimate bleeding risk in mild bleeding disorders. J Thromb Haemost 2007;5 (Suppl 1):157-166

13 Rodeghiero F, Tosetto A, Abshire T, et al; ISTH/SSC Joint VWF and Perinatal/Pediatric Hemostasis Subcommittees Working Group. ISTH/SSC bleeding assessment tool: a standardized questionnaire 
and a proposal for a new bleeding score for inherited bleeding disorders. J Thromb Haemost 2010;8(09):2063-2065

14 Hayward CP, Moffat KA, Plumhoff E, Van Cott EM. Approaches to investigating common bleeding disorders: an evaluation of North American coagulation laboratory practices. Am J Hematol 2012; 87(Suppl 1):S45-S50

15 Al-Samkari H, Connors JM. Managing the competing risks of thrombosis, bleeding, and anticoagulation in patients with malignancy. Hematology (Am Soc Hematol Educ Program) 2019; 2019(01):71-79

16 Menegatti M, Biguzzi E, Peyvandi F. Management of rare acquired bleeding disorders. Hematology (Am Soc Hematol Educ Program) 2019;2019(01):80-87

17 Manco-Johnson MJ, Grabowski EF, Hellgreen M, et al. Laboratory testing for thrombophilia in pediatric patients. On behalf of the Subcommittee for Perinatal and Pediatric Thrombosis of the Scientific and Standardization Committee of the International Society of Thrombosis and Haemostasis (ISTH). Thromb Haemost 2002;88(01):155-156

18 Merriman L, Greaves M. Testing for thrombophilia: an evidencebased approach. Postgrad Med J 2006;82(973):699-704

19 Hayward CP, Webert KE. Expert approaches to common bleeding and thrombotic problems-part I. Semin Thromb Hemost 2012;38 (07):641-644

20 Middeldorp S. Is thrombophilia testing useful? Hematology (Am Soc Hematol Educ Program) 2011;2011:150-155

21 Bruce A, Massicotte MP. Thrombophilia screening: whom to test? Blood 2012;120(07):1353-1355

22 Holzhauer S, Goldenberg NA, Junker R, et al. Inherited thrombophilia in children with venous thromboembolism and the familial risk of thromboembolism: an observational study. Blood 2012; 120(07):1510-1515

23 Barbosa ACN, Montalvão SAL, Barbosa KGN, et al. Prolonged APTT of unknown etiology: a systematic evaluation of causes and laboratory resource use in an outpatient hemostasis academic unit. Res Pract Thromb Haemost 2019;3(04):749-757

24 Bux-Gewehr I, Morgenschweis K, Zotz RB, Budde U, Scharf RE. Combined von Willebrand factor deficiency and factor XII deficiency. Thromb Haemost 2000;83(03):514-516

25 Tiede A, Scharf RE, Dobbelstein C, Werwitzke S. Management of acquired haemophilia A. Hamostaseologie 2015;35(04): 311-318

26 Tang N, Yu F, Cao W, Li D. Significantly prolonged PT and APTT could indicate a wide spectrum of clinical manifestations in three patients with plasma cell disorders. Int J Lab Hematol 2019;41 (01):e23-e26

27 Manning SC, Beste D, McBride T, Goldberg A. An assessment of preoperative coagulation screening for tonsillectomy and adenoidectomy. Int J Pediatr Otorhinolaryngol 1987;13(03):237-244

28 Kussmann I, Koller M, Heinke T, Rothmund M. [Value of preoperative blood coagulation analysis for assessment of hemorrhage risk in general surgery]. Chirurg 1997;68(07):684-688

29 Asaf T, Reuveni H, Yermiahu T, et al. The need for routine preoperative coagulation screening tests (prothrombin time PT/ partial thromboplastin time PTT) for healthy children undergoing elective tonsillectomy and/or adenoidectomy. Int J Pediatr Otorhinolaryngol 2001;61(03):217-222

30 Johnson RK, Mortimer AJ. Routine pre-operative blood testing: is it necessary? Anaesthesia 2002;57(09):914-917

$31 \mathrm{Kim} \mathrm{KN}$, You WC. The value of preoperative coagulation screening in children undergoing surgery. Korean J Hematol 2008;43:98-105

32 McHugh J, Holt C, O'Keeffe D. An assessment of the utility of unselected coagulation screening in general hospital practice. Blood Coagul Fibrinolysis 2011;22(02):106-109

33 Houry S, Georgeac C, Hay JM, Fingerhut A, Boudet MJThe French Associations for Surgical Research. A prospective multicenter evaluation of preoperative hemostatic screening tests. Am J Surg 1995;170(01):19-23
34 Seicean A, Schiltz NK, Seicean S, Alan N, Neuhauser D, Weil RJ. Use and utility of preoperative hemostatic screening and patient history in adult neurosurgical patients. J Neurosurg 2012;116 (05):1097-1105

35 Zaher G, Al-Noury K. The value of routine preoperative testing in the prediction of operative hemorrhage in adenotonsillectomy. Indian J Otolaryngol Head Neck Surg 2014;66(Suppl 1):30-36

36 Koscielny J, Ziemer S, Radtke $\mathrm{H}$, et al. A practical concept for preoperative identification of patients with impaired primary hemostasis. Clin Appl Thromb Hemost 2004;10(03):195-204

37 Scharf RE. Acquired platelet function defects: an underestimated but frequent cause of bleeding complications in clinical practice. In: Scharf RE, ed. Progress and Challenges in Transfusion Medicine, Hemostasis, and Hemotherapy. Basel: Karger; 2008: 296-316

38 Scharf RE. Acquired disorders of platelet function. In: Michelson AD, Cattaneo M, Frelinger AL, Newman PJ, eds. Platelets. 4th ed. Cambridge, MA: Academic Press; 2019:905-920

39 Favaloro EJ. The utility of the PFA-100 in the identification of von Willebrand disease: a concise review. Semin Thromb Hemost 2006;32(05):537-545

40 Scharf RE. Drugs that affect platelet function. Semin Thromb Hemost 2012;38(08):865-883

41 Scharf RE. Management of bleeding in patients using antithrombotic agents: prediction, prevention, protection and problemoriented intervention. Hamostaseologie 2009;29(04):388-398

42 Colucci G, Alberio L, Damarmels Biasiutti F, Lämmle B. Bilateral periorbital ecchymoses. An often missed sign of amyloid purpura. Hamostaselogie 2014;34(03):249-252

43 Nicoleau A, Nicoleau CA. Images in clinical medicine. Hereditary hemorrhagic telangiectasia (Osler-Weber-Rendu disease). N Engl J Med 1999;340(23):1800

44 Pérez-Belmonte LM, Gomez-Moyano E. Images in clinical medicine: Osler-Weber-Rendu Syndrome. N Engl J Med 2015;373(13): e15

45 Pfanner G, Koscielny J, Pernerstorfer T, et al; Austrian Society for Anaesthesia, Resuscitation and Intensive Care. [Preoperative evaluation of the bleeding history. Recommendations of the working group on perioperative coagulation of the Austrian Society for Anaesthesia, Resuscitation and Intensive Care]. Anaesthesist 2007;56(06):604-611

46 Albert FW, Eichler $\mathrm{H}$, Haubelt $\mathrm{H}$, et al; für das Kompetenznetz Hämostaseologie Rheinland-Pfalz-Saarland. [Haemostatic testing prior to elective surgery? Yes!]. Hamostaseologie 2009;29(01): 58-63

47 Bidlingmaier C, Eberl W, Knöfler R, Olivieri M, Kurnik K. Haemostatic testing prior to elective surgery in children? Not always!. Hamostaseologie 2009;29(01):64-67

48 Fritsch G, Flamm M, Hepner DL, Panisch S, Seer J, Soennichsen A. Abnormal pre-operative tests, pathologic findings of medical history, and their predictive value for perioperative complications. Acta Anaesthesiol Scand 2012;56(03):339-350

49 Böhmer AB, Wappler F, Zwissler B. Preoperative risk assessmentfrom routine tests to individualized investigation. Dtsch Arztebl Int 2014;111(25):437-445, quiz 446

50 Chee YL, Crawford JC, Watson HG, Greaves MBritish Committee for Standards in Haematology. Guidelines on the assessment of bleeding risk prior to surgery or invasive procedures. $\mathrm{Br} \mathrm{J}$ Haematol 2008;140(05):496-504

51 Cosmi B, Alatri A, Cattaneo M, et al; Italian Society for Haemostasis and Thrombosis. Assessment of the risk of bleeding in patients undergoing surgery or invasive procedures: guidelines of the Italian Society for Haemostasis and Thrombosis (SISET). Thromb Res 2009;124(05):e6-e12

52 Bonhomme F, Ajzenberg N, Schved JF, Molliex S, Samama CM; French Anaesthetic and Intensive Care Committee on Evaluation of Routine Preoperative Testing French Society of Anaesthesia and Intensive Care. Pre-interventional haemostatic assessment: 
Guidelines from the French Society of Anaesthesia and Intensive Care. Eur J Anaesthesiol 2013;30(04):142-162

53 Anderson DR, Morgano GP, Bennett C, et al. American Society of Hematology 2019 guidelines for management of venous thromboembolism: prevention of venous thromboembolism in surgical hospitalized patients. Blood Adv 2019;3(23):3898-3944

54 O'Brien SH. Bleeding scores: are they really useful? Hematology (Am Soc Hematol Educ Program) 2012;2012:152-156

55 Rydz N, James PD. The evolution and value of bleeding assessment tools. J Thromb Haemost 2012;10(11):2223-2229

56 Bonhomme F, Boehlen F, Clergue F, de Moerloose P. Preoperative hemostatic assessment: a new and simple bleeding questionnaire. Can J Anaesth 2016;63(09):1007-1015

57 Fasulo MR, Biguzzi E, Abbattista M, et al. The ISTH Bleeding Assessment Tool and the risk of future bleeding. J Thromb Haemost 2018;16(01):125-130

58 Vries MJ, van der Meijden PE, Kuiper GJ, et al. Preoperative screening for bleeding disorders: A comprehensive laboratory assessment of clinical practice. Res Pract Thromb Haemost 2018;2 (04):767-777

59 Kemkes-Matthes B. A pleading: comprehensive clinical information is mandatory for appropriate hemostasis laboratory testing. Hamostaseologie (in revision)

60 Wuillemin WA. Point-of-care testing: Vollblutmethoden in der Hämostasediagnostik. Hamostaseologie 2000;20:13-16

61 Spannagl M, Dick A, Junker R. POCT in coagulation. Quality assurance. Hamostaseologie 2010;30(02):82-90

62 Sucker C, Zotz RB, Senft B, et al. Exercise-induced hemostatic alterations are detectable by rotation thrombelastography (ROTEM): a marathon study. Clin Appl Thromb Hemost 2010; 16(05):543-548

63 Perry DJ, Fitzmaurice DA, Kitchen S, Mackie IJ, Mallett S. Point-ofcare testing in haemostasis. Br J Haematol 2010;150(05):501-514
64 Seidel H, Rahman MM, Scharf RE. Monitoring of antiplatelet therapy. Current limitations, challenges, and perspectives. Hamostaseologie 2011;31(01):41-51

65 Weber CF, Zacharowski K. Perioperative point of care coagulation testing. Dtsch Arztebl Int 2012;109(20):369-375

66 Lier H, Vorweg M, Hanke A, Görlinger K. Thromboelastometry guided therapy of severe bleeding. Essener Runde algorithm. Hamostaseologie 2013;33(01):51-61

67 Paniccia R, Priora R, Liotta AA, Abbate R. Platelet function tests: a comparative review. Vasc Health Risk Manag 2015;11:133-148

68 Zhi M, Ding EL, Theisen-Toupal J, Whelan J, Arnaout R. The landscape of inappropriate laboratory testing: a 15-year metaanalysis. PLoS One 2013;8(11):e78962

69 Bindraban RS, Ten Berg MJ, Naaktgeboren CA, Kramer MHH, Van Solinge WW, Nanayakkara PWB. Reducing test utilization in hospital settings: a narrative review. Ann Lab Med 2018;38 (05):402-412

70 Sarkar MK, Botz CM, Laposata M. An assessment of overutilization and underutilization of laboratory tests by expert physicians in the evaluation of patients for bleeding and thrombotic disorders in clinical context and in real time. Diagnosis (Berl) 2017;4(01):21-26

71 GTH News. Erfolgreicher Launch der GTH Mediathek im Juni. Hamostaseologie 2020;40(03):382

72 GTH News. Early career research grants of the Society of Thrombosis and Hemostasis Research. Hamostaseologie 2020;40:383

73 McLintock C, Pabinger I, Bauer KA, et al. International Society on Thrombosis and Haemostasis core curriculum project: core competencies in clinical thrombosis and hemostasis. J Thromb Haemost 2016;14(01):3-27

74 Moffat KA, Kiencke V, Blanco AN, et al. International Society on Thrombosis and Haemostasis core curriculum project: core competencies in laboratory thrombosis and hemostasis. J Thromb Haemost 2019;17(11):1848-1859 